Genetic analyses of Seoul hantavirus genome recovered from rats (Rattus norvegicus) in the Netherlands unveils diverse routes of spread into Europe

\title{
Ling, Jiaxin
}

2019-05

Ling , J , Verner-Carlsson , J , Eriksson , P , Plyusnina, A, Loehmus , M , Jaerhult , J D , van de Goot , F , Plyusnin , A , Lundkvist , A \& Sironen , T 2019 , ' Genetic analyses of Seoul hantavirus genome recovered from rats (Rattus norvegicus) in the Netherlands unveils diverse routes of spread into Europe ' , Journal of Medical Virology , vol. 91 , no. 5 , pp. 724-730 . https://doi.org/10.1002/jmv.25390

http://hdl.handle.net/10138/313503

https://doi.org/10.1002/jmv.25390

unspecified

publishedVersion

Downloaded from Helda, University of Helsinki institutional repository.

This is an electronic reprint of the original article.

This reprint may differ from the original in pagination and typographic detail.

Please cite the original version. 


\title{
Genetic analyses of Seoul hantavirus genome recovered from rats (Rattus norvegicus) in the Netherlands unveils diverse routes of spread into Europe
}

\author{
Jiaxin Ling ${ }^{1}$ | Jenny Verner-Carlsson ${ }^{1}$ | Per Eriksson ${ }^{1}{ }^{10}$ | Angelina Plyusnina ${ }^{2}$ | \\ Mare Löhmus $^{1,3,4,5}$ | Josef D. Järhult ${ }^{6}$ | Frank van de Goot ${ }^{7}$ | Alexander Plyusnin ${ }^{1,2}$ | \\ Åke Lundkvist $^{1}{ }^{\text {(i) }}$ | Tarja Sironen ${ }^{2}$
}

${ }^{1}$ Department of Medical Biochemistry and Microbiology, Zoonosis Science Center, University of Uppsala, Uppsala, Sweden

${ }^{2}$ Department of Virology, Medicum, University of Helsinki, Helsinki, Finland

${ }^{3}$ Institute for Environmental Medicine, Karolinska Institutet, Stockholm, Sweden

${ }^{4}$ Centre for Occupational and Environmental Medicine, Stockholm County Council, Stockholm, Sweden

${ }^{5}$ The National Veterinary Institute, Uppsala, Sweden

${ }^{6}$ Section for Infectious Diseases, Department of Medical Sciences, Uppsala University, Uppsala, Sweden

${ }^{7}$ Symbiant Pathology Expert Centre, Alkmaar, The Netherlands

\section{Correspondence}

Åke Lundkvist, Department of Medical Biochemistry and Microbiology, Zoonosis Science Center, University of Uppsala, Uppsala 582, 75123, Sweden.

Email: ake.lundkvist@imbim.uu.se

Funding information

The Swedish Research Council, Grant/Award Number: 2017-05807; Vetenskapsrådet
Seoul virus (SEOV) is the etiologic agent of hemorrhagic fever with renal syndrome. It is carried by brown rats (Rattus norvegicus), a commensal rodent that closely cohabitates with humans in urban environments. SEOV has a worldwide distribution, and in Europe, it has been found in rats in UK, France, Sweden, and Belgium, and human cases of SEOV infection have been reported in Germany, UK, France, and Belgium. In the search of hantaviruses in brown rats from the Netherlands, we found both serological and genetic evidence for the presence of SEOV in the local wild rat population. To further decipher the relationship with other SEOV variants globally, the complete genome of SEOV in the Netherlands was recovered. SEOV sequences obtained from three positive rats (captured at close trapping locations at the same time) were found highly similar. Phylogenetic analyses demonstrated that two lineages of SEOV circulate in Europe. Strains from the Netherlands and UK, together with the Baxter strain from US, constitute one of these two, while the second includes strains from Europe and Asia. Our results support a hypothesis of diverse routes of SEOV spread into Europe. These findings, combined with other indications on the expansion of the spatial European range of SEOV, suggest an increased risk of this virus for the public health, highlighting the need for increased surveillance.

KEYWORDS

hantavirus, hemorrhagic fever with renal syndrome, phylogenetic analysis, Seoul virus

\section{1 | INTRODUCTION}

The hantaviruses constitute the genus Orthohantavirus in the family Hantaviridae, order Bunyavirales. Like the other members of this order, the hantaviruses are negative-strand RNA viruses with a genome divided in three segments: large (L), medium (M), and small (S), which encode the viral RNA-dependent RNA-polymerase, the precursor for surface glycoproteins $\mathrm{Gn}$ and $\mathrm{Gc}$, and the nucleocapsid protein (N), respectively. ${ }^{1}$ In some hantaviruses, the $\mathrm{S}$ segment encodes also for a nonstructural protein. $^{2}$

The hantaviruses are carried by rodents, bats, and insectivores. ${ }^{3}$ When transmitted to humans, some hantaviruses cause either of the two clinical syndromes: hemorrhagic fever with renal syndrome (HFRS), or hantavirus (cardio) pulmonary syndrome. ${ }^{4}$ The geographical distribution of hantaviruses follows that of their specific natural reservoirs with which the viruses remain tightly associated. ${ }^{3,5}$ Because of the various natural reservoirs, the different groups of 
hantaviruses are found on different continents: for example, Hantaan virus (HTNV), associated with the striped field mouse (Apodemus agrarius), occurs in Asia (China, the Far east of Russia, Korea, and the neighbouring countries), while Sin Nombre virus carried by the deer mouse (Peromyscus manuculatus) is found only in North America. ${ }^{4}$ The sole exception to this pattern is Seoul virus (SEOV), the only cosmopolitan hantavirus found worldwide (Eurasia, Africa, and America), together with its host, the commensal, or Norway, rat (Rattus norvegicus). ${ }^{6}$ It is thought that SEOV originated in China, and was subsequently exported to Europe and later spread through the New World following human migrations and sea-trade. ${ }^{6}$ In Europe, SEOV has been found in wild rats in the UK, ${ }^{7}$ France, ${ }^{8,9}$ and Belgium, ${ }^{10}$ and in pet rats in France, Sweden, and UK. ${ }^{11-13}$ SEOV is causing HFRS, which is generally somewhat milder than the disease caused by HTNV, but more severe than Puumala virus (PUUV) infection. ${ }^{14}$ SEOV infections are also found associated with hepatitis. ${ }^{15} \mathrm{~A}$ recent imported human case of SEOV infection has been reported in Germany. ${ }^{16}$ HFRS cases in laboratory workers in Belgium, the Netherlands, UK, and France have been recorded, ${ }^{17-20}$ and human SEOV cases have been found in the UK and France. ${ }^{13,21}$

In the Netherlands, circulation of two hantaviruses in wild rodents has been reported; PUUV in bank voles (Myodes glareolus), and Tula virus (TULV) in common voles (Microtus arvalis). ${ }^{22}$ In 2013 , rats trapped by the regional water authority in Rijn and IJsselland in the Eastern province of Gelderland in the Netherlands were found to be SEOV antibody positive. These samples had been confirmed by pan- $L$ reverse transcription polymerase chain reaction (RT-PCR). ${ }^{23}$ The present study expanded on the previous work, by subjecting the positive samples to complete SEOV genome sequencing. The aim of this study was to recover SEOV genome sequences from wild rats trapped in the Netherlands, and analyse their relationships with other SEOV variants from Europe and elsewhere.

\section{2 | MATERIALS AND METHODS}

\section{1 | Rodent sampling}

Sixteen rats were collected by the regional water authority in Rijn and IJsselland in the eastern province of Gelderland in the Netherlands in 2013 as previously reported. ${ }^{23}$ Species, sex, and age of the collected rodents were recorded. The animals were dissected and samples of blood were used for the serological tests, while the lung samples were stored at $-85^{\circ} \mathrm{C}$ until further genetic analysis.

\section{2 | Reverse transcription-polymerase chain reaction for detection of hantaviral genomes}

RNA was extracted from the lung tissue samples using TRIzol (Life Technologies, Carlsbad, CA) according to the manufacturer's instruction. The RNA was tested for the presence of hantaviral genomes by the widely used pan-hanta-L-segment method. ${ }^{24}$ RNA from positive samples was subjected to RT-PCR to recover complete sequences of hantaviral $\mathrm{L}, \mathrm{M}$, and $\mathrm{S}$ segments. PCR was performed in $20 \mu \mathrm{L}$ reaction mixtures using the Phusion Flash High-Fidelity PCR Master Mix (Thermo Fisher Scientific, Lithuania). PCR-products were separated by electrophoresis on $1.5 \%$ agarose gels and purified using the QIAQuick Gel Extraction Kit (Qiagen, Hilden, Germany). Oligonucleotide primers that cover the complete coding regions and partial noncoding regions were designed by using all SEOV sequences for 13 amplifications of the genome (Supporting InformationTable S1).

Once hantavirus positive samples were confirmed, total DNA from rodent tissue samples was prepared using TRIzol. The cytochrome b (cyt-b) gene of mitochondrial DNA (mtDNA) was amplified by universal primers as previously described for the rodent species confirmation. ${ }^{25}$

\section{3 | Sequencing}

PCR amplicons were purified using the QIAquick PCR Purification kit (Qiagen). All PCR amplicons from a given individual were pooled together. Sequencing libraries were prepared (1 $\mathrm{ng}$ of input DNA) using the Nextera XT DNA library prep kit (Illumina, San Diego, CA) according to the manufacturer's instruction. Sequencing was performed on an Illumina MiSeq Instrument using the MiSeq reagent kit version 2 (300-cycles) (Illumina). Assembly of the sequence data was performed using the CLC genome workbench with the SEOV strain Humber as the reference sequence. ${ }^{7} \mathrm{~A}$ total of 60 to $80 \%$ of recoverage of SEOV sequences has been obtained and the gaps were closed by PCRs. The obtained SEOV sequences have been submitted to GenBank; accession numbers are MG972931-MG972939.

\section{4 | Genetic analysis}

The datasets were built by using the new sequences from the current study and representative SEOV strains retrieved from Genbank. ${ }^{10,26}$ Sequences were aligned (in codons) using MAFFT (https://mafft.cbrc.jp/ alignment/software/) with default settings followed by manual refinement. In total, two datasets have been used in this study. One set contained the coding regions and was used for the genetic analysis; the other data set included the complete sequencing regions, and was used for the phylogenetic analysis. The potential recombination events were sought using the Phi-test in SPLITS TREE 4.0. ${ }^{27}$

Model optimizations were calculated for each data set, as followed by the calculation of pairwise genetic distances between the SEOV strains by using MEGA $7 .{ }^{28}$ All genetic distance matrices were analyzed and visualized with $R$ Studio $(R)^{29}$ by using ggplot function.

\section{5 | Phylogenetic analysis}

Trees were reconstructed using IQ-TREE program (http://iqtree.cibiv. univie.ac.at/) after selection of the best-fit substitution model. ${ }^{30,31}$ The branch support values were assessed by 1000 ultrafast bootstrap pseudoreplicates and SH-like approximate likelihood tests. ${ }^{32,33}$ Finally, trees were viewed and edited using the FigTree v1.4.2 software (http:// tree.bio.ed.ac.uk/software/figtree/). 


\section{3 | RESULTS}

\section{1 | Confirmed circulation of SEOV in Dutch rats}

Out of sixteen rats, three screened positive for antibodies to hantavirus by enzyme-linked immunosorbent assay, indirect immunofluorescence assay, and immunoblotting, which was reported in a previous study. ${ }^{23}$ In this study, we aimed to recover the genome of SEOV by using RT-PCR. We tested these three rats by using Pan-L RT-PCR and they were positive for SEOV RNA; these were the same individuals in which specific anti-SEOV antibodies were previously found. ${ }^{23}$ Corresponding wild-type (wt) SEOV strains (that have not been isolated in cell culture), obtained from these three individuals, were designated as SEOV/Gelderland/ Rn22/2013, SEOV/Gelderland/Rn33/2013, and SEOV/Gelderland/Rn84/ 2013, or Gelderland22, Gelderland33, and Gelderland84, for short. Cyt-b sequences obtained from these three samples confirmed the host species as Rattus norvegicus.

\section{2 | Genetic analysis}

First, partial hantavirus $L$ segment sequences of 330 nucleotides ( $n t)$ were recovered for these three strains: Gelderland22, Gelderland33, and Gelderland84, and they were all identical. We further recovered $6437 \mathrm{nt}$ for the L segment sequence of Gelderland84. For the S segment, we were able to recover 1697,1321 , and $1503 \mathrm{nt}$ for the Gelderland22, Gelderland33, and Gelderland84, respectively. For all three strains, the $\mathrm{N}$ protein-encoding open reading frame (ORF) was found to be $1290 \mathrm{nt}$ long (corresponding to positions 43-1332 of the complete S-segment sequence of Humber, Genbank Acc. No. KM948598), encoding a putative $N$ protein of 429 amino acids (aa) in length. For the $M$ segment, we succeeded in determining 3503 , 3521, and $3598 \mathrm{nt}$ for the Gelderland22, Gelderland33, and Gelderland84 strains, respectively. The M-segment had a single ORF encoding a putative GPC protein of 1134 aa residues. A putative signal peptide of 17 aa in the beginning of the ORF, and the 642WAASA656 motif determining the cleavage of GPC into the 641Gn (aa) and 487Gc (aa) glycoproteins were identified as well.

All three Dutch strains appeared very close to each other, the $S$ and partial $L$ segment sequences (330 nt) were identical. The $M$ segment sequences showed $0.1 \%$ differences, compared with Gelderland84, there were only single nucleotide changes at positions 1944 and 2439 of Gelderland22 and Gelderland33, respectively.

For the L segment, the Dutch SEOV showed the highest sequence identity with the strains Humber and Cherwell originating from the UK: $98 \%$ nt ( $99 \%$ aa) and $96 \%$ nt (99\% aa), respectively, and with the isolate IR461 (from the laboratory outbreak in the UK) $96 \% \mathrm{nt}$ ( $97 \%$ aa). Compared to the other SEOV variants from Europe, the identity was 90 to $96 \%$ nt $(98-100 \%$ aa) to the French strains LYO903, GIV726, TURCKHEIM/Hu/FRA/2016 (Genbank accession nos: KJ502300, KJ502303, and KX064268), 93\% nt (100\% aa) to the Belgian strain SEO/Belgium/Rn895/2005 (Genbank accession NO. JQ898108), and $92 \%$ nt $(100 \%$ aa) to the Swedish strain Rn1466 (Genbank accession NO. KY688131). Compared to the closest hantavirus relatives, the Dutch SEOV showed identities of $62 \% \mathrm{nt}$ (84\% aa), and 62\% nt (83\% aa) to the Serang (Genbank accession NO. AM998806) and Thailand viruses (Genbank accession NO. JN116261), respectively.

To visualize the genetic relatedness of the Gelderland84 strain (for which we obtained the longest sequences), the $S$ and $M$ segment sequence identities were plotted against other reference SEOV strains worldwide (Supporting Information Figure S1). As expected, for both the $S$ and $M$ segments, the Dutch SEOV strains showed the highest sequence identity with the strains originating from the UK. The genetic distances of the SEOV strains were observed to have somewhat geographic relatedness: the SEOV strains within the continent of Asia (especially the Chinese strains) were closer than between the different continents.

To further analyse the diversity of SEOV from different continents, the pairwise genetic distances of the different segments were calculated and compared (Figure 1). For the S segment, since there were only two strains available from the Americas, the genetic distance of SEOV was compared only between Europe and Asia, and the difference was not statistically significant as determined by the Mann-Whitney test (data not shown). Also for the M segment, the genetic distance of SEOV from the three regions (Europe, Asia,
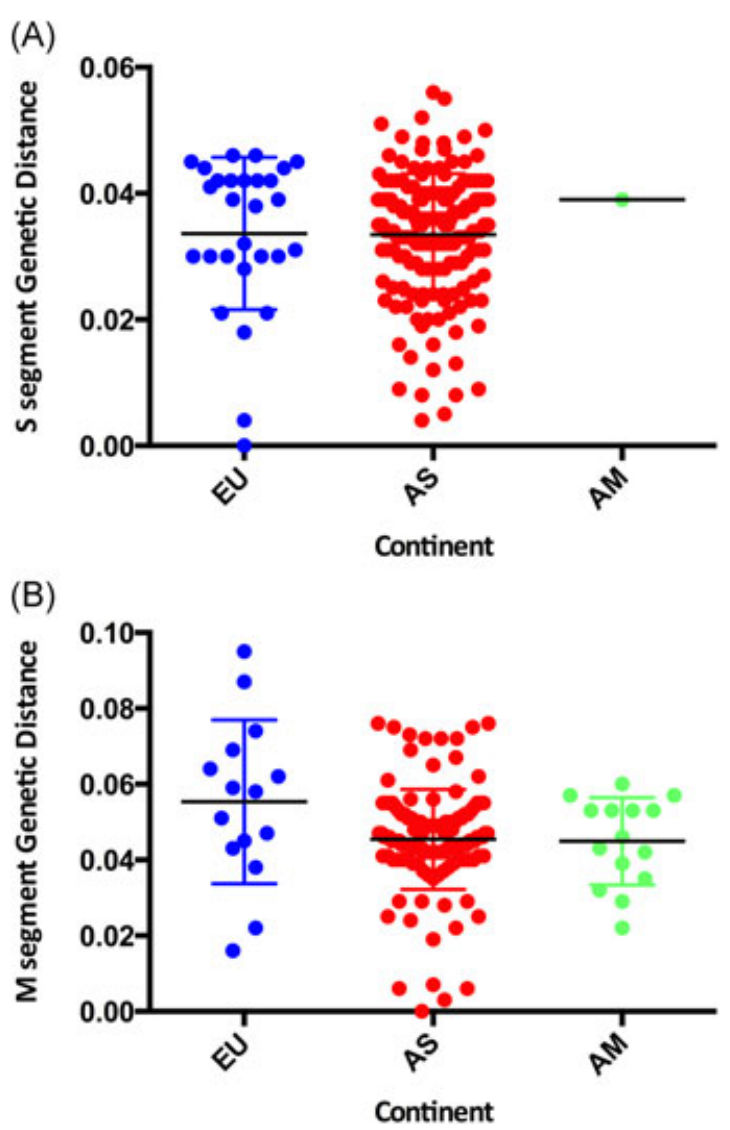

FIGURE 1 Genetic diversity of the entire coding regions of $S(A)$ and $M(B)$ segments of SEOV grouped in different continents. The colour indicators are: red for Asia (AS), green for America (AM), and blue for Europe (EU). SEOV, Seoul virus 
and America) was not significantly different according to the Kruskal-Walllis test (data not shown).

\subsection{Phylogenetic analysis}

Recombination events were not observed in any of the three alignments ( $P$-values of Phi test were over 0.05), hence all three alignments were used for phylogenetic analyses. Figure 2 (A-C) shows the existence of nine distinct, well-supported, and genetic lineages of SEOV worldwide, with similar topology to that described earlier ${ }^{10,34}$; numbered as lineages \#1 to \#4 and \#6 to \#9, and the lineage \#5 being the genetically distinct Gou virus (GOUV). Lineages \#1 and \#3 included most SEOV strains from China (provinces Wan, Shanxi, Henan, Hubei and others), while the strains from Wuhan and Jiangxi, China, were outside of the lineages \#1 and \#3. ${ }^{35,36}$ SEOV lineage \#2, contained also the Houston strain from USA. Lineage \#4 consisted of strains from South Korea (Seoul 80-39, the prototype SEOV strain), USA (strains Tchoupitoulas and New Orleans), and China (North-eastern area). Lineage \#6 included strains from Africa (strain Egypt) and USA (strains Girard Point and Philadelphia). Lineage \#7 contained strains from Europe (France, Belgium, and Sweden) and from South-East Asia: Indonesia, Singapore, Vietnam, and Cambodia. Lineage \#8 included strains $\mathrm{KI}$ from Japan and the lineage \#9 contained strains from Europe (UK: Humber and Cherwell), the Baxter strain from New York, and strains from Baltimore and Brazil. Not surprisingly, on the S-, M-, and L-phylogenetic trees, the newly described Dutch SEOV strains clustered in closest proximity to each other and, together with strains Humber and Cherwell, formed a well-supported lineage.

The SEOV strains showed clear geographic patterns, as we could find only Asian SEOV strains from lineages \#5, \#1, \#7, and \#3. As a result, the extant SEOV strains worldwide might be originated from China or at least the Asian continent, which is in line with earlier observations. ${ }^{6}$ This Asian origin is also supported by the ancestral position of GOUV (Figure 2). In the American continent, SEOV strains have been found in lineages \#2, \#4, \#6, and \#9, grouped with other SEOV strains from Asia or Europe. In Europe, two distinct lineages \#9 and \#7 are present, and SEOV strains from these two lineages cocirculate in France. In the other European countries, such as the Netherlands, UK, Belgium, and Sweden, SEOV showed even more strict geographic clustering by the presence of only one lineage, suggesting that France might have served as the important locations for exchanging SEOV strains in Europe. However, due to the limited amount of data, the exact routes still remain obscure.

\section{DISCUSSION}

SEOV has a worldwide geographic distribution, from Asia to Africa, Europe, and America. In Europe, the genetic evidence of SEOV has been found in UK first in a laboratory outbreak, ${ }^{37,38}$ and consequently in France, ${ }^{9,13}$ Belgium, ${ }^{10,39}$ and Sweden. ${ }^{11}$ In the current study, we recovered the full genome sequences of Dutch SEOV by using multiplex PCR combined with next generation sequencing techniques, similar to the method described by Kim et al. ${ }^{34}$ Our result showed that SEOV strains from the lineage \#9 are circulating among the wild brown rats in the Netherlands.

The characterization of Dutch SEOV strains showed a closer genetic relatedness to SEOV strains from UK than to the other SEOV strains worldwide. This distribution of SEOV in Europe and globally today might be the result of virus-host codiversification and the migrations of the host, R. norvegicus. ${ }^{6}$ Like other hantaviruses, especially Muridae-borne ones, SEOV is coevolving with its host. ${ }^{40}$ $R$. norvegicus originated either in northern China or in Mongolia, ${ }^{41,42}$ and emerged in Europe only in the 1500s. In the 1750s, R. norvegicus was introduced in North America. ${ }^{43}$ These distributions have been strongly influenced by human activities and movement between different continents. During this process, the colonization of $R$. norvegicus into the new continent has been well established, and so has SEOV, which has been carried by rats. In the phylogenetic tree of SEOV, we observed that lineage \#9, comprising of SEOV from US, UK, France, and Netherlands, was segregated from the other lineages, with a comparatively longer branch length, further suggesting that the diversification of this lineage is older than the other extant lineages. Lineage \#7 of SEOV is also circulating in Europe, and comprises SEOV strains from France, Belgium, and Sweden, and they are all close to SEOV strains from southeast Asia; including Indonesia, Singapore, Cambodia, and Vietnam. This suggests multiple introductions of SEOV into Europe, and discrete and independently evolving foci in the local regions. Interestingly, none of the currently known Chinese SEOV strains from lineages \#1, \#3, and \#5 has been found in Europe, even with more frequent human activities, or trades between Europe and Asia nowadays. One possible explanation for this is that recruitment of new populations of $R$. norvegicus is limited at the present time, i.e., during the postcolonization of brown rats. ${ }^{44}$ This is also supported by the observation of similar diversity of SEOV in different continents, indicating that diversification rates in America, Asia, and Europe are comparable.

In our study, we have recovered SEOV genomes from three positive rats and the sequences were found highly similar. This is probably because the rats were all captured in close trapping locations and at the same time. In Europe, we observed the circulation of two lineages, \#7 and \#9, but only lineage \#9 has been found in the Netherlands. Since lineage \#7 contains strains from Asia, this suggests a more recent transmission from Asia to Europe. However, the surveillance effort on SEOV has clearly been insufficient, and further data is needed to trace the spread of SEOV.

SEOV is responsible for a milder form of HFRS as compared to the disease caused by HTNV, with a case-fatality rate less than $1 \% .4$ However, some SEOV-associated HFRS human cases still need hospitalization. ${ }^{13,16,46}$ The transmission of SEOV to humans usually occurs through direct contact with infected rodents. Several instances of human SEOV infection in Europe have been described. The first one (strain IR461) was a strain isolated from a rat in an outbreak of multiple cases in laboratory animal technicians and seems also to have been 

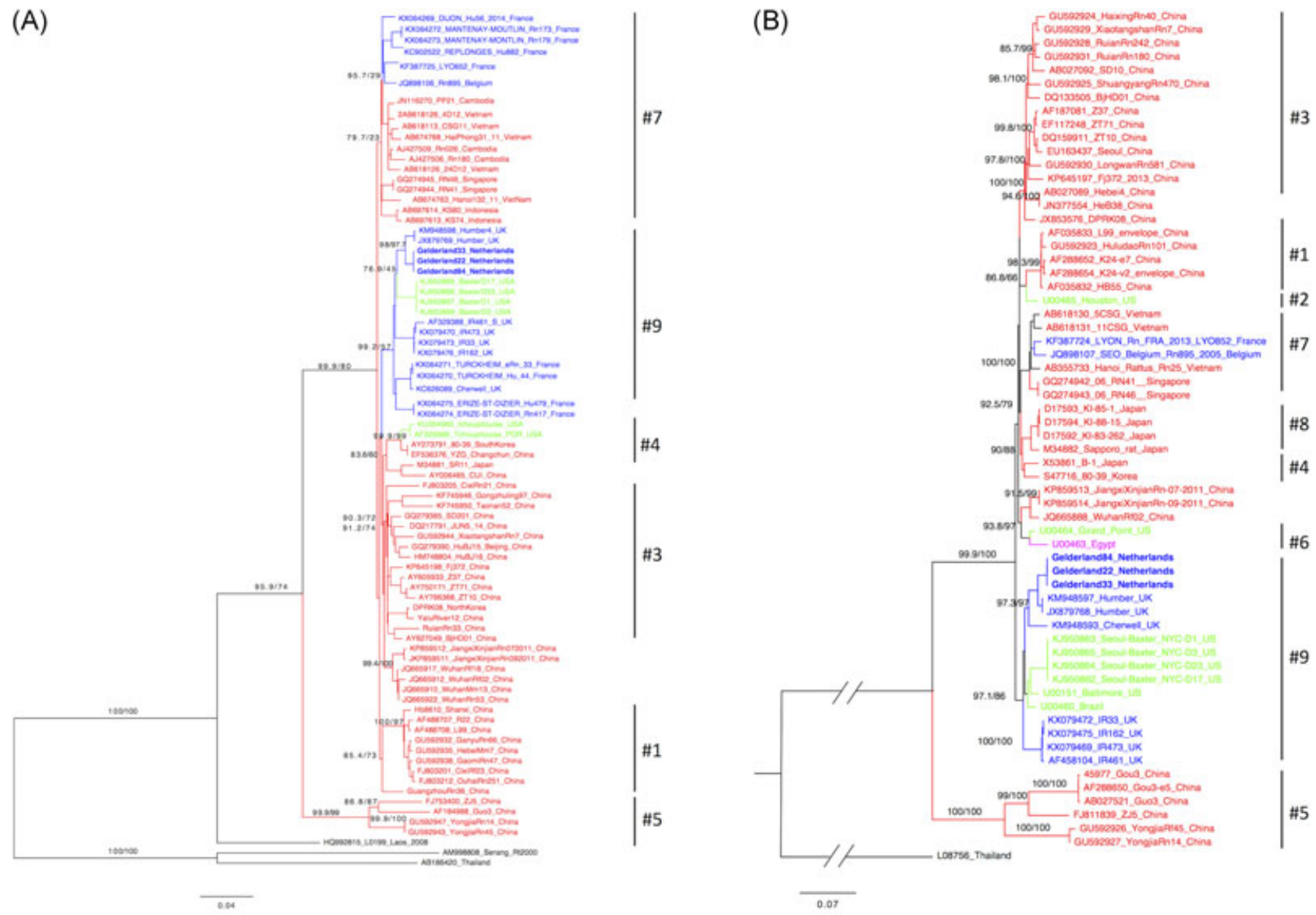

(C)

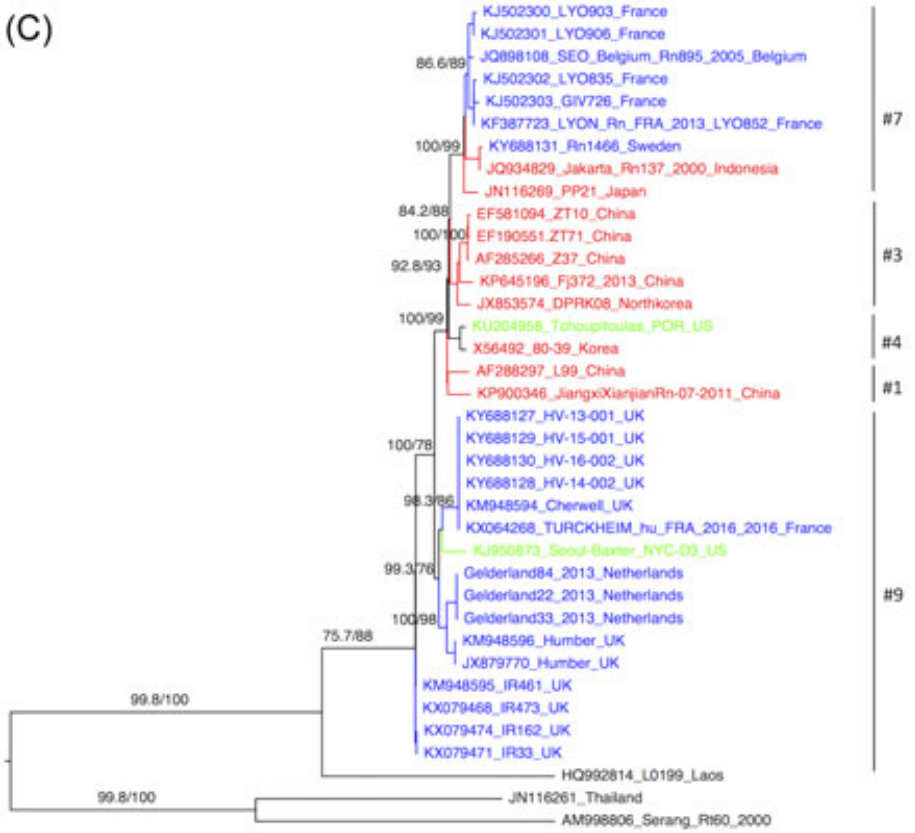

0.2

FIGURE 2 IQ trees based on the partial S-(A), M-(B), and L segment (C) of SEOV. The phylogenetic tree was generated by IQ trees, using the best-fit GTR $+\mathrm{G}+r$ model of evolution as estimated from the data by the jModel test, based on the alignment of the $\mathrm{S}-(1600 \mathrm{nt})$, the $\mathrm{M}-(3600 \mathrm{nt})$, and L-(6400-nt) segments. The ultrafast bootstrap values and the SH-like approximate likelihood values are shown on the branches. The scale bar means the nucleotide substitutions per site. The colour indicators are red for Asia (AS), green for America (AM), blue for Europe (EU), and purple for Africa (AF). SEOV, Seoul virus 
linked to similar cases in other European institutions. ${ }^{38}$ Later on, two nonlaboratory human infections have also been reported in Europe: a farmer in UK and a pregnant woman in France. ${ }^{47,48}$ Recently, an outbreak of HFRS caused by SEOV among rat owners, breeders, and distributors of the pet animal market was reported in UK. ${ }^{12,21,49}$ Most cases were occupational exposure related and confirmed by specific anti-SEOV IgG detection. In addition, acute human SEOV infections in France have been reported by detecting SEOV-specific IgM and viral RNA (from lineages \#7 and \#9). ${ }^{13}$ In US, SEOV has been detected in city rats in New York. ${ }^{50}$ In 2017, outbreaks of SEOV infections occurred in several states of US. ${ }^{51}$ This indicated that SEOV infections might be underdiagnosed, especially by the physicians outside of Asia. In the Netherlands, until very recently, a human case of SEOV infection has been reported. ${ }^{46}$ Although serology and RT-PCR for a broad panel of both Cricetidae-borne and Muridae-borne hantaviruses are performed in the Netherlands for more than five years, underdiagnosis is suspected due to unawareness among physicians. ${ }^{52}$

In conclusion, at least two different lineages of SEOV are circulating in either wild or pet rats in Europe as demonstrated by detections in UK, France, Belgium, Sweden, and Netherlands. SEOV may also be found in other European countries. This latest finding of SEOV from Dutch rats confirms a potential threat for the public health and highlights the need for further studies.

\section{ACKNOWLEDGEMENTS}

This study was supported by Vetenskapsrådet (Swedish Research Council, 2017-05807), We thank Dr. Chantal Reusken and Dr. Johan Reimerink from the Netherlands National Institute for Public Health and the Environment (RIVM) for valuable comments on the manuscript.

\section{ORCID}

Per Eriksson (D) http://orcid.org/0000-0001-7633-403X

Åke Lundkvist (D) http://orcid.org/0000-0001-8608-6551

\section{REFERENCES}

1. Group. TIBS. Create a new order, Bunyavirales, to accommodate nine families (eight new, one renamed) comprising thirteen genera. ICTV; 2016.

2. Hepojoki J, Strandin T, Lankinen H, Vaheri A. Hantavirus structure-molecular interactions behind the scene. J Gen Virol. 2012;93(Pt 8): 1631-1644.

3. Plyusnin A, Sironen T. Evolution of hantaviruses: co-speciation with reservoir hosts for more than 100 MYR. Virus Res. 2014;187:22-26.

4. Jonsson CB, Figueiredo LTM, Vapalahti O. A global perspective on hantavirus ecology, epidemiology, and disease. Clin Microbiol Rev. 2010;23(2):412-441.

5. Bennett SN, Gu SH, Kang HJ, Arai S, Yanagihara R. Reconstructing the evolutionary origins and phylogeography of hantaviruses. TIM. 2014;22(8):473-482.

6. Lin XD, Guo WP, Wang W, et al. Migration of Norway rats resulted in the worldwide distribution of Seoul hantavirus today. J Virol. 2012;86(2):972-981.
7. Jameson $\mathrm{LJ}$, Logue $\mathrm{CH}$, Atkinson $\mathrm{B}$, et al. The continued emergence of hantaviruses: isolation of a Seoul virus implicated in human disease, United Kingdom, October 2012. Euro Surveill. 2013;18(1):4-7.

8. Dupinay T, Pounder KC, Ayral F, et al. Detection and genetic characterization of Seoul virus from commensal brown rats in France. Virol J. 2014;11:32.

9. Heyman P, Plyusnina A, Berny P, et al. Seoul hantavirus in Europe: first demonstration of the virus genome in wild Rattus norvegicus captured in France. Eur J Clin Microbiol Infect Dis. 2004;23(9):711-717.

10. Plyusnina A, Heyman P, Baert K, Stuyck J, Cochez C, Plyusnin A. Genetic characterization of seoul hantavirus originated from norway rats (Rattus norvegicus) captured in Belgium. J Med Virol. 2012;84(8): 1298-1303.

11. Lundkvist Å, Verner-Carlsson J, Plyusnina A, Forslund L, Feinstein R, Plyusnin A. Pet rat harbouring Seoul hantavirus in Sweden, June 2013. Euro Surveill. 2013;18(27):15-18.

12. Mcelhinney LM, Marston DA, Pounder KC, et al. High prevalence of Seoul hantavirus in a breeding colony of pet rats. Epidemiol Infect. 2017;145(15):3115-3124.

13. Reynes JM, Carli D, Bour JB, et al. Seoul virus infection in humans, France, 2014-2016. Emerg Infect Dis. 2017;23(6):973-977.

14. Krüger $\mathrm{DH}$, Ulrich $\mathrm{R}$, Lundkvist Å. Hantavirus infections and their prevention. Microbes Infect. 2001;3(13):1129-1144.

15. Kim YS, Ahn C, Han JS, Kim S, Lee JS, Lee PW. Hemorrhagic fever with renal syndrome caused by the Seoul virus. Nephron. 1995;71(4): 419-427.

16. Hofmann J, Weiss S, Kuhns M, Zinke A, Heinsberger H, Kruger DH. Importation of human Seoul virus infection to Germany from Indonesia. Emerg Infect Dis. 2018;24(6):1099-1102.

17. Heyman P, Baert K, Plyusnina A, et al. Serological and genetic evidence for the presence of Seoul hantavirus in Rattus norvegicus in Flanders, Belgium. Scand J Infect Dis. 2009;41(1):51-56.

18. Groen J, Jordans HGM, Clement JPG, et al. Identification of Hantavirus serotypes by testing of post-infection sera in immunofluorescence and enzyme-linked immunosorbent assays. J Med Virol. 1991;33(1):26-32.

19. Groen J, Gerding MN, Jordans JGM, Clement JP, Nieuwenhuijs JHM, Osterhaus ADME. Hantavirus infections in The Netherlands: epidemiology and disease. Epidemiol Infect. 1995;114(2):373-383.

20. Osterhaus AD, Spijkers I, van Steenis G, van der Groen G. [Hantavirus infections in the Netherlands]. Ned Tijdschr Geneeskd. 1984;128(52): 2461-2462.

21. Duggan JM, Close R, McCann L, et al. A seroprevalence study to determine the frequency of hantavirus infection in people exposed to wild and pet fancy rats in England. Epidemiol Infect. 2017;145(12):2458-2465.

22. Reusken C, de Vries A, Adema J, et al. First genetic detection of Tula hantavirus in wild rodents in the Netherlands. J Infection. 2008;57(6): 500-503.

23. Verner-Carlsson J, Lõhmus $M$, Sundström K, et al. First evidence of Seoul hantavirus in the wild rat population in the Netherlands. Infect Ecol Epidemiol. 2015;5:27215.

24. Klempa B, Fichet-Calvet E, Lecompte E, et al. Hantavirus in African wood mouse, Guinea. Emerg Infect Dis. 2006;12(5):838-840.

25. Ling J, Sironen T, Voutilainen L, et al. Hantaviruses in Finnish soricomorphs: evidence for two distinct hantaviruses carried by Sorex araneus suggesting ancient host-switch. Infect Genet Evol. 2014;27:51-61.

26. Wang H, Yoshimatsu K, Ebihara $\mathrm{H}$, et al. Genetic diversity of hantaviruses isolated in china and characterization of novel hantaviruses isolated from Niviventer confucianus and Rattus rattus. Virology. 2000;278(2):332-345.

27. Huson DH, Bryant D. Application of phylogenetic networks in evolutionary studies. Mol Biol Evol. 2006;23(2):254-267.

28. Kumar S, Stecher G, Tamura K. MEGA7: molecular evolutionary genetics analysis version 7.0 for bigger datasets. Mol Biol Evol. 2016; 33(7):1870-1874. 
29. Team. RDC R: A language and environment for statistical computing. $\mathrm{R}$ Foundation for Statistical Computing, Vienna, Austria. ISBN 3-900051-07-0, URL http://www.r-project.org/. In:2008.

30. Trifinopoulos J, Nguyen LT, Von haeseler A, Minh BQ. W-IQ-TREE: a fast online phylogenetic tool for maximum likelihood analysis. Nucleic Acids Res. 2016;44(W1):W232-W235.

31. Kalyaanamoorthy S, Minh BQ, Wong TKF, von Haeseler A, Jermiin LS. ModelFinder: fast model selection for accurate phylogenetic estimates. Nat Methods. 2017;14(6):587-589.

32. Minh BQ, Nguyen MAT, von Haeseler A. Ultrafast approximation for phylogenetic bootstrap. Mol Biol Evol. 2013;30(5):1188-1195.

33. Guindon S, Dufayard JF, Lefort V, Anisimova M, Hordijk W Gascuel O. New algorithms and methods to estimate maximumlikelihood phylogenies: assessing the performance of PhyML 3.0. Syst Biol. 2010;59(3):307-321.

34. Kim WK, No JS, Lee SH, et al. Multiplex PCR-based next-generation sequencing and global diversity of Seoul virus in humans and rats. Emerg Infect Dis. 2018;24(2):249-257.

35. Kang YJ, Zhou DJ, Tian JH, et al. Dynamics of hantavirus infections in humans and animals in Wuhan city, Hubei, China. Infect Genet Evol. 2012;12(8):1614-1621.

36. Liu J, Liu DY, Chen W, et al. Genetic analysis of hantaviruses and their rodent hosts in central-south China. Virus Res. 2012;163(2): 439-447.

37. Shi X, McCaughey C, Elliott RM. Genetic characterisation of a hantavirus isolated from a laboratory-acquired infection. J Med Virol. 2003;71(1):105-109.

38. Lloyd G, Bowen ETW, Jones N, Pendry A. HFRS outbreak associated with laboratory rats in UK. Lancet. 1984;1(8387): 1175-1176.

39. Desmyter J, Johnson KM, Deckers C, Leduc JW, Brasseur F, Van ypersele de strihou C. Laboratory rat associated outbreak of haemorrhagic fever with renal syndrome due to Hantaan-like virus in Belgium. Lancet. 1983;2(8365-66):1445-1448.

40. Plyusnin A, Morzunov SP. Virus evolution and genetic diversity of hantaviruses and their rodent hosts. Curr Top Microbiol Immunol. 2001;256:47-75.

41. Song Y, Lan Z, Kohn MH. Mitochondrial DNA phylogeography of the Norway rat. PLoS One. 2014;9(2):e88425.

42. Smith AT, Xie Y. A guide to the mammals of China. Princeton, NJ: Princeton University Press; 2008;143-160.

43. Armitage P. Commensal rats in the New World, 1492-1992. Biologist. 1993;40:174-178.
44. Puckett EE, Park J, Combs M, et al. Global population divergence and admixture of the brown rat (Rattus norvegicus). Proc Biol Sci. 2016;283(1841):20161762.

45. Zhang YZ, Zou Y, Fu ZF, Plyusnin A. Hantavirus infections in humans and animals, China. Emerg Infect Dis. 2010;16(8):1195-1203.

46. Swanink C, Reimerink J, Gisolf J, et al. Autochthonous Human Case of Seoul Virus Infection, the Netherlands. Emerg Infect Dis. 2018;24(12): 2158-2163.

47. Adams K, Jameson L, Meigh R, Brooks T. Hantavirus: an infectious cause of acute kidney injury in the UK. BMJ Case Rep. 2014;2014 bcr2014205529.

48. Mace G, Feyeux C, Mollard N, et al. Severe Seoul hantavirus infection in a pregnant woman, France, October 2012. Euro Surveill. 2013; 18(17):20464.

49. McElhinney L, Fooks AR, Featherstone C, Smith R, Morgan D. Hantavirus (Seoul virus) in pet rats: a zoonotic viral threat. Vet Rec. 2016;178(7):171-172.

50. Firth C, Bhat M, Firth MA, et al. Detection of zoonotic pathogens and characterization of novel viruses carried by commensal Rattus norvegicus in New York City. mBio. 2014;5(5):e01933-01914.

51. Kerins JL, Koske SE, Kazmierczak J, et al. Outbreak of Seoul virus among rats and rat owners-United States and Canada, 2017. MMWR Morb Mortal Wkly Rep. 2018;67(4):131-134.

52. Goeijenbier M, Verner-Carlsson J, van Gorp EC, et al. Seoul hantavirus in brown rats in the Netherlands: implications for physicians--epidemiology, clinical aspects, treatment and diagnostics. Neth J Med. 2015;73(4):155-160.

\section{SUPPORTING INFORMATION}

Additional supporting information may be found online in the Supporting Information section at the end of the article.

How to cite this article: Ling J, Verner-Carlsson J, Eriksson P, et al. Genetic analyses of Seoul hantavirus genome recovered from rats (Rattus norvegicus) in the Netherlands unveils diverse routes of spread into Europe. J Med Virol. 2019;91:

724-730. https://doi.org/10.1002/jmv.25390 